\title{
Pathways of Migrants and Refugees - a Simulation Approach
}

\author{
Felix Breitenecker ${ }^{1}$, Tamara Vobruba ${ }^{1}$, Andreas Körner ${ }^{1}$, Nikolas Popper ${ }^{2}$
}

${ }^{1}$ TU Wien, Inst. f. Analysis und Scientific Computing, Mathematical Modelling Group,

Wiedner Hauptstrasse 8-10, 1040 Vienna, Austria

${ }^{2}$ dwh Simulation Services, Neustiftgasse 57-59, 1070 Vienna, Austria; *felix.breitenecker@tuwien.ac.at

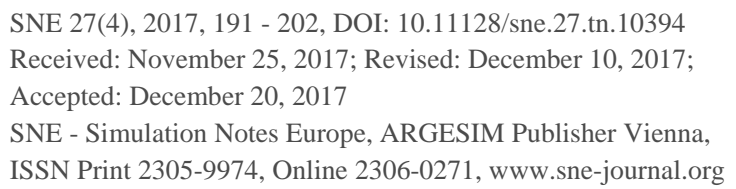

SNE 27(4), 2017, 191 - 202, DOI: 10.11128/sne.27.tn.10394 Received: November 25, 2017; Revised: December 10, 2017; Accepted: December 20, 2017

SNE - Simulation Notes Europe, ARGESIM Publisher Vienna, ISSN Print 2305-9974, Online 2306-0271, www.sne-journal.org

Abstract. In autumn 2015, Europe was confronted with a dramatic migration and refugee's movement - the so-called Refugee Crisis 2015. No ebbing of the migration and refugee pathways can be expected for the near future. Can simulation help to analyse the pathway of migration in such a critical case, and can simulation forecast pathways of migrants and refugees under different circumstances? This contribution first sketches variants of the Spatial Interaction Model, a model approach for any kind of population shift in certain regions. This more theoretical introduction is followed by the development of a Spatial Interaction Model for the pathway of migrants and refugees during the Refugee Crisis 2015, and by model implementation and identification of model parameters based on UNHCR data reported during the crisis.
Next follows a spatial extension including the Mediterranean Sea route allowing a forecast and real data comparison for pathways of migrants and refugees in summer 2016. Last step is development of a Scenario Model allowing the study of possible control actions. The simulation model does not provide direct help for the people involved, but the simulation can help in better understanding and improving the situation of migrants and refugees - the authors hope.

\section{Introduction}

In September and October 2015 a wave of refugees shocked Europe - later on called Refugee Crisis 2015. UNHCR reported daily dramatic data - refugees on route from Syria to western Europe, mainly on transit in Eastern Europe, and applying for asylum in Western Europe. Figure 1 shows two snapshots from animations of UNHCR data- the one from September 5, 2015, when the wave started, and the other from October 30, 2015, when the wave ebbed down.
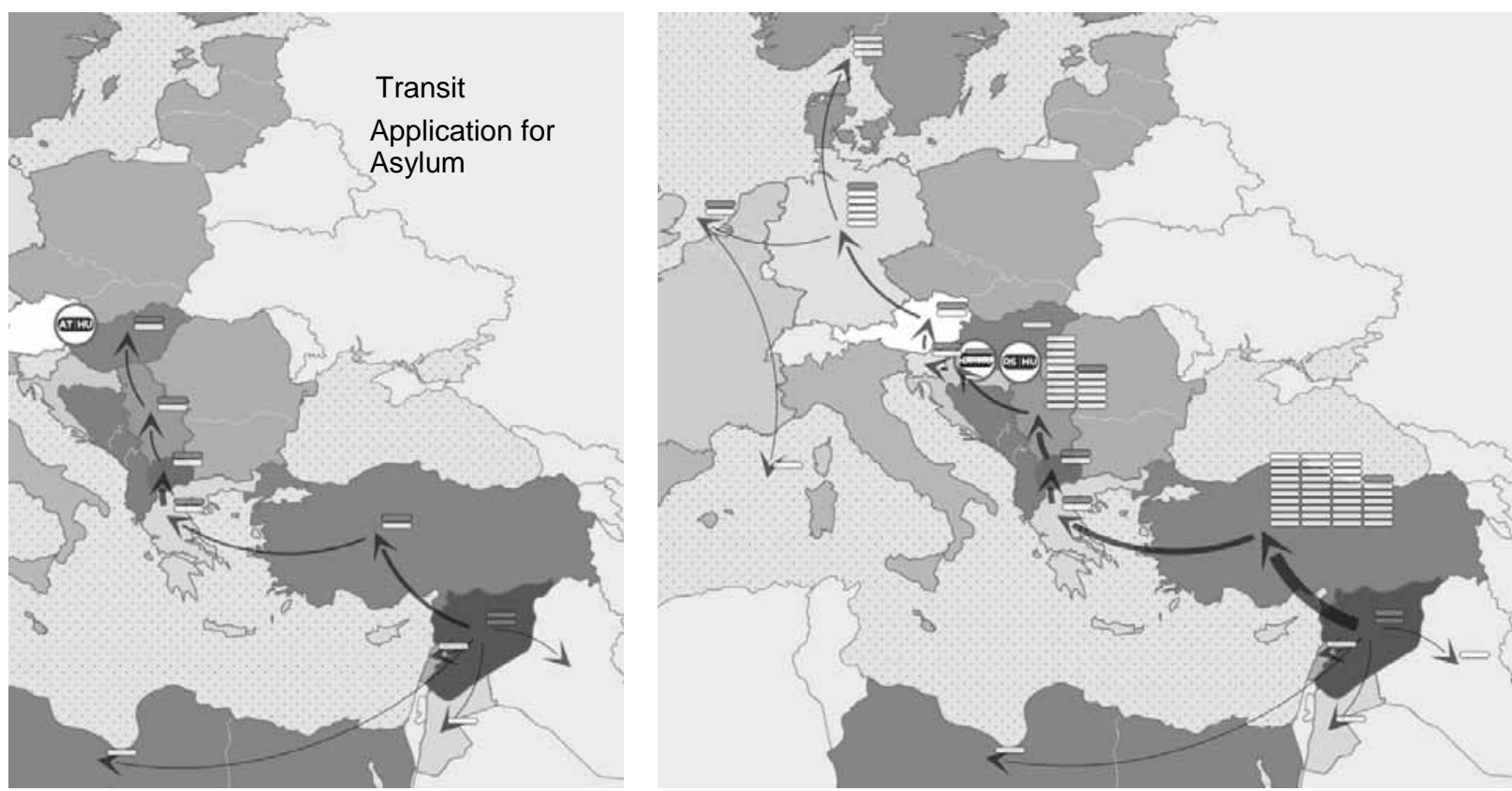

Figure 1. Data animation of refugees' pathway in Refugee Crisis 2015 - snapshot 2015-09-05 (left) and 2015-10-30 (right). 
The data animation of this specific refugee wave (two snapshots in Figure 1) was performed by $d w h$ Simulation Services for ORF 1 Online (Austrian Broadcast Company, Science Program Online). The Mathematical Modelling Group of the Institute for Analysis and Scientific Computing at TU Wien is closely co-operating with $d w h$ Simulation Services, and discussions on the Refugee Crisis 2015 initiated a small project for trying to develop a simulation model for this specific refugee wave, with possibilities to parametrize for other possible pathways of refugees or migrants.

First step was the interpretation of a refugee wave as a special case of migration behaviour. Migration behaviour seems to be driven by individual and personal circumstances of the refugees. Moreover, it becomes clear from a macroscopic viewpoint, that migration is a special kind of spatial population dynamics. Parts of a population migrate from one region into another region, driven by repulsive forces in the start region, and by attractive forces of the destination region(s). Regions often try to control migration by border control - separation forces for the migration movement. These forces, together with the pathway of migration, allow a modelling approach for the migration movement as behavioural spatial interaction model.

The pathways of migrants and refugees can be interpreted as spacial interaction of parts of populations in certain regions. The so-called Spatial Interaction Model is a macroscopic model approach for describing any kind of spatial interaction behaviour between populations or regions (described in Section 1 Spatial Interaction Models). This modelling approach has a wide range of applications, from traffic flows, movement of commuters or migrants, trade of goods or transmission of messages - and therefore seems to be appropriate for modelling also the pathway of migrants and refugees.

The best known and widely used type of Spatial Interaction Models is the Social Gravity Model. (sketched in Section 2 Social Gravity Models). The basic idea is the description of the interaction between populations or regions with a relation referring to Newton's law of gravity.

Section 3 Migration Pathway Model develops a network of generalized social gravity models for the pathway of migrants and refugees, taking into account special demands with respect to regions of origin, possible regions of destination and the transit regions, and with respect to the heterogeneity of the attraction or repulsion of these regions influencing the migration behaviour. It turns out that it is necessary to introduce transit countries, characterized by an attraction not from the country itself, but by the attraction of following countries.

Indeed, in spring 2016, the developed model could be parametrized and identified for the refugee wave of the Refugee Crisis 2015 - with specific submodels for the attracting and repelling attributes of the involved countries, and with data from UNHCR (Section 4 Refugee Crisis Model).

Obviously, the Refugee Crisis 2015 was not the only refugee wave. In general, no ebbing of the migration and refugee pathways can be expected, and the challenge was, whether the developed model is able to forecast the refugee wave expected for June 2016. Section 5 Forecast Model describes the necessary model changes - close down of Balkan route and opening of Mediterranean route - and a successful forecast.

It turns out, that the model - first published and presented in autumn 2016 [7, 8] is 'general' enough to study qualitatively also certain possible control strategies, proposed by politicians, with more or less careful considerations - close-down of certain specific borders, etc. briefly sketched in Section 6 Scenario Model.

A summary in Section 7 tries to evaluate the developed model. Main conclusions is: Models are in any case a simplification of reality, but they should help in better understanding of complex dynamics as migration movement, and the intention of this model is to show strategies to improve the situation of migrant population and resident population under appropriate prerequisites.

\section{Spatial Interaction Models}

Spatial interaction is a movement or transmission over space between at least two regions, which is resulting of a decision process involving different influences. Interaction as physical movement is for example migration movement, where nonphysical movement could be the transmission of messages or the exchange of knowledge.

The Spatial Interaction Model of two regions (Figure 2 ) is describing such spatial interaction with a relation, which is depending on different attributes. In the most general form, this model is given by the following equation:

$$
I_{i, j}=f\left(A_{i}, R_{j}, C_{i, j}\right)
$$




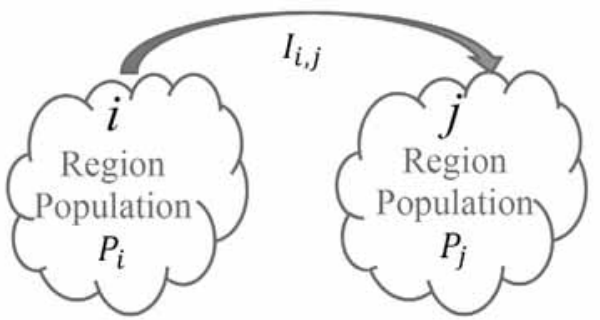

Figure 2. Population movement between regions.

The interaction factor $I_{i, j}$ ('Immigration'), responsible for movement from population in region $i$ to population in region $j$, is described by a function $f$ of attracting attributes $A_{i}$ in region $i$, of repelling attributes $R_{j}$ in region $j$ and separation attributes $C_{i, j}$ between region $i$ and region $j$ (Figure 2).

The interaction is depending on a decision process, which is happening based on certain conditions. For example, the decision of a commuter for a certain traffic route could be influenced by the costs of this route, the availability of public transport and the distance to work. These influencing factors are represented by attributes.

The shift of population is then given by $I_{i, j} \cdot P_{i}$, so that a time-dependent dynamics is introduced:

$$
\begin{aligned}
& P_{j, \text { next }}=P_{j, \text { prev }}+I_{i, j} \cdot P_{i, \text { prev }} \\
& P_{i, \text { next }}=P_{i, \text { prev }}-I_{i, j} \cdot P_{i, \text { prev }}
\end{aligned}
$$

\section{Social Gravity Models}

The widely used type of Spatial Interaction Models are the (Social) Gravity Models. Here the relation, which is describing the interaction, is based on Newton's law of gravity. This idea to draw analogies between physics and certain human behaviour has a long history. It came up in the year 1852 by Henry Charles Carey, who described the human migration behaviour as the 'tendency to gravitate the fellow man' [1]. In geography and demography, Gravity Models are used for a long time to analyse the flow of people, goods or capital. But often there is a lack of mathematical and theoretical foundation to understand the backgrounds ([2]).

This leads to examination of the relation between migration movement and the attraction of regions, as well as the distance between regions. Ernest Charles Young was the first who postulated the formal connection with the law of gravity [9]. Investigating the movement of farm population, he pointed out this coherence in the following formula:

$$
M=k \cdot \frac{F}{D^{2}}
$$

Here $M$ is the absolute migration, $F$ the intensity of attraction of a region, $D$ the distance to this region and $k$ a proportional constant. The dependency of attraction and the distance is based on Newton's law of gravity.

In the following years, this approach was used to describe human shopping behaviour and was refined by John Quincy Steward in the year 1941, who was developing the theory of demographic gravitation ([6]):

$$
I_{i, j}=G \cdot \frac{P_{i} \cdot P_{j}}{d_{i, j}^{2}}
$$

Here the influence of the fundamental physical law is obvious. The interaction $I_{i, j}$ between the population centres $i$ and $j$ is direct proportional to the product of the socalled population masses $P_{i}$ and $P_{j}$, which are describing the attributes in centres $i$ and $j$.

Furthermore the interaction is indirect proportional to the squared distance $d_{i, j}^{2}$ between the population centres $i$ and $j$. The constant $G$ is called the demographic gravity constant.

A few years later in 1950 Steward developed a formula, which involves the possibility of different impacts of the attributes to the interaction ([6]):

$$
I_{i, j}=G \cdot \frac{w_{i} P_{i} \cdot w_{j} P_{j}}{d_{i, j}^{2}}
$$

Here $w_{i}$ and $w_{j}$ are population weights. These weights are reflecting the heterogeneity of population masses and are treated as statistical parameters. With this parametric extension, the model description steps back from the direct analogy to Newton's law, but it increases the flexibility of the mode a lot.

After the introduced forms of gravity models, many different formalisations were used. Ashish Sen and Tony Smith were introducing a general class of gravity models ([6]).

The general class of gravity models has the following form

$$
I_{i, j}=A(i) \cdot B(j) \cdot F\left(d_{i, j}\right)
$$

where the interaction $I_{i, j}$ is resulting of the product of a weighted function $A(i)$ of attributes in $i$, a weighted function of the attributes $B(j)$ in $j$ and function of separation attributes $F\left(d_{i, j}\right)$ between $i$ and $j$. These weighted functions are vector-valued, so they can include a set of different attributes and heterogeneity.

SNE 27(4) - 12/2017 


\section{Migration Pathway Model}

Next aim is to develop a migration model with the pathway (network) of migration, which is fitting to the introduced class of gravity models. Therefore, the focus is on the migration behaviour between the regions of origin, the possible regions of destination and the transit regions.

The heterogeneity of the attraction or repulsion of these regions influencing the migration behaviour should be included. Furthermore, this attribute function should be treated as time dependent to observe structural changes in the migration behaviour over time. The model description should also have the flexibility to describe migration movement between a list of different included regions in different structure and through different routes.

\subsection{Graph model for pathway}

Directed graphs are a generic approach for describing connected regions and possible pathways of migration. Here, the graph of migration movement is defined as directed graph $G=(V, E)$, where the finite set of vertices

$$
V(G)=\left\{v_{1}, v_{2}, \ldots, v_{l}\right\}
$$

is describing the different regions of interest and the finite set of edges

$$
E(G)=\left\{e_{1}, e_{2}, \ldots, e_{k}\right\} \text { with } e_{r}=\left\langle v_{i}, v_{j}\right\rangle
$$

is describing the geographical possibility to migrate from one region $v_{i}$ to the other region $v_{j}$ (in the adjacency matrix of the graph denoted by a 1 ).

\subsection{Discrete model on pathway}

With this formalism, it is possible to describe a timedependent interaction $I_{i, j}(t)$ from region $v_{i}$ to region $v_{j}$ (Figure 3 ) with the following equation:

$$
I_{i, j}(t)=\frac{\sum_{k=1}^{n} a_{k} \cdot\left(A_{j}(t)\right)_{k} \cdot \sum_{k=1}^{m} r_{k} \cdot\left(R_{i}(t)\right)_{k}}{\sum_{k=1}^{n} c_{k} \cdot\left(C_{i, j}(t)\right)_{k} \cdot}
$$

If $M_{i}(t)$ and $M_{j}(t)$ denote the number of migrants in regions $v_{i}$ or $v_{j}$ resp., then the number of migrants $M_{i, j}(t)$ moving from region $v_{i}$ to region $v_{j}$ is given by

$$
M_{i, j}(t)=I_{i, j}(t) \cdot M_{i}(t)
$$

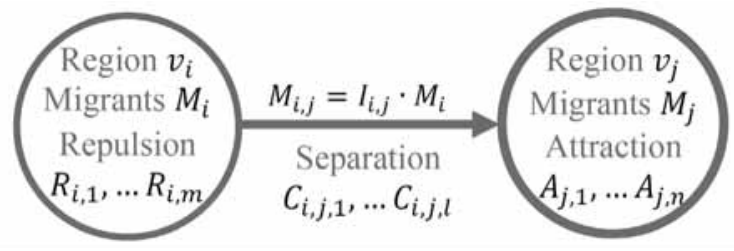

Figure 3. Migration movement between regions with attributes for attraction, repulsion, and separation.

Here the $n$ functions of attractive attributes $A_{j}$

$$
\left(A_{j}(t)\right)_{k=1}^{n}:[0, T] \rightarrow[0,1], a_{k} \in[0,1], \sum_{1}^{n} a_{k}=1
$$

in region $v_{j}$ are time-dependent, are normalized to 1 and are weighted by parameters $a_{k}$, which are the basis for following model identification. These properties also belong to the $m$ functions of repulsive attributes $R_{i}$ in region $v_{i}$

$$
\left(R_{i}(t)\right)_{k=1}^{m}:[0, T] \rightarrow[0,1], r_{k} \in[0,1], \sum_{1}^{m} r_{k}=1
$$

For the $l$ functions of separation attributes $C_{i, j}$

$$
\left(C_{i, j}(t)\right)_{k=1}^{l}:[0, T] \rightarrow[0, \infty), c_{k} \in[0,1], \sum_{1}^{l} c_{k}=1
$$

another value range is foreseen, in order to describe closed borders.

In general, time base for migration movement can be different - decades, years, quarters, months, and days. In any case, a synchronous time base results in a discrete dynamic model for migration from the region $v_{i}$ to region $v_{j}$, using the actual migration equation with the interaction factor $M_{i, j}(t)=I_{i, j}(t) \cdot M_{i}(t)$ :

$$
\begin{aligned}
& M_{j}(t+1)=M_{j}(t)+I_{i, j}(t) \cdot M_{i}(t) \\
& M_{i}(t+1)=M_{i}(t)-I_{i, j}(t) \cdot M_{i}(t)
\end{aligned}
$$

Clearly, any region may have more than one neighbour with immigration and emigration, so that in the above equations, more bilance terms will appear, but structurally only neighbouring regions are taken into account:

$M_{j}(t+1)=M_{j}(t)+M_{i, j}(t)+\sum M_{r, j}(t)-\sum M_{j, s}(t)$
$M_{i}(t+1)=M_{j}(t)-M_{i, j}(t)-\sum M_{u, i}(t)+\sum M_{i, v}(t)$ In above equations, the sums spread over neighbouring emigration regions and immigration regions, except region $v_{i}$ and region $v_{j}$, which are directly balanced in the equations. 


\subsection{Discrete model with transit regions}

The consideration of only neighbouring regions in the up to now developed model may be sufficient for regions, which all are potential immigration regions. Especially in the case of refugee waves, some regions are only transit regions, which have high repelling attributes and which must have attractive attributes; but these attractive attributes are not a property of the regions itself, they are given by the attractive properties of the immigration neighbours of the transit region.

This fact requires a model extension for the calculation of attractive attributes of a transit region $v_{j}-$ using the attractive attributes of the neighbouring immigration regions $v_{j_{1}}, v_{j_{2}}, \ldots v_{j_{u}}$ given by

$$
A_{j_{r}}^{*}=\sum_{k=1}^{n} a_{k} \cdot\left(A_{j_{r}}(t)\right)_{k}
$$

Instead of the generic summed up attractive attributes $A_{j}^{*}$, the maximum of summed up attractive attributes of neighbouring immigration regions is used:

$$
A_{j}^{+}(t)=\max _{u=j_{1}, \ldots, j_{u}} A_{u}^{*}(t)
$$

Also the separation attributes $C_{j, j_{r}}^{*}$ from transit region $v_{j}$ to the neighbouring immigration regions $v_{j_{1}}, v_{j_{2}}, \ldots v_{j_{u}}$ must be taken into account - one appropriate solution is to make use of separated maximum calculation:

$$
A_{j}^{+}(t)=\max _{u=j_{1}, \ldots, j_{u}}\left(A_{u}^{*}(t) \cdot \max _{v=j_{1}, \ldots, j_{u}} C_{j, v}^{*}(t)\right)
$$

Additionally also the repulsion attributes $R_{j}^{*}(t)$ are increased to $R_{j}^{*}(t)$ depending on a certain transit category. Figure 4 sketches these extensions for transit.

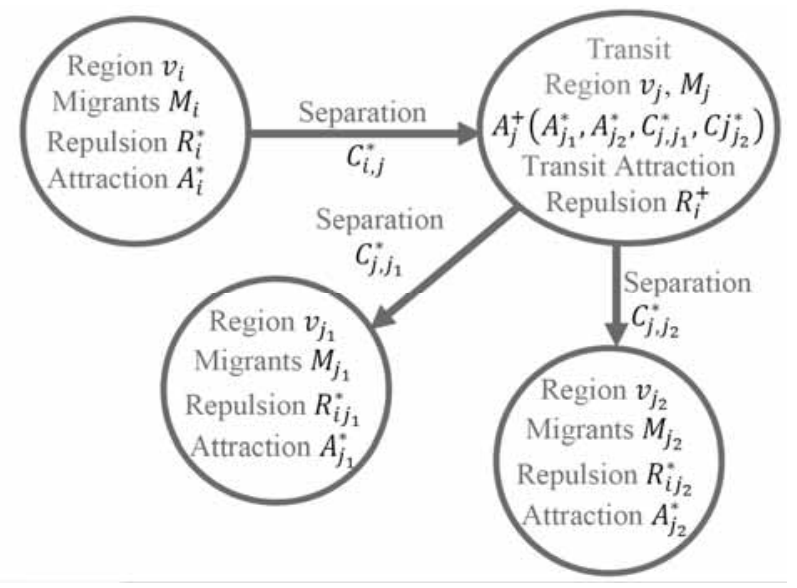

Figure 4. Migration movement through a transit region with dependent attraction attributes.

\subsection{Relation to social behaviour modelling}

Since the presented discrete model is simulating social behaviour, it must be mentioned, that the social aspects and reasons behind the migration are a result of a complex composition of various influences. Finding appropriate descriptions for social behaviour is a great challenge, and the available different approaches are suited to cover the different aspects, but not the overall behaviour. As consequence, the characterization of the presented migration model is important in terms of understanding the qualities and limits of this approach.

Empirical studies showed that gravity models are most successful in describing macroscopic patterns of spatial interaction. They are more reliable in picturing the behaviour of populations rather than individuals ([6]). One reason is that individual decisions are often influenced by many different partly unknown factors, which refer to specific individuals. Here, agent-based modelling may have better merits, but less features for quantitative identification. On the other hand, the focus on population groups gives the quality of a sufficient description of the behaviour of interest, with just a little required information.

It is also important to discuss the aspect of time. The dynamics of the introduced migration model are only observed at discrete time steps, staying constant over the next period of time. Structural changes, for example a change of the attribute function from one time step to the next one, are 'treated' as event. From this viewpoint, the model shows quasi-static behaviour, depending on the chosen time base.

The model is by mathematical definition a deterministic model, but most of the parameters are rates - averages of probabilistic distributions. From this viewpoint, the model is averaging probabilistic behaviour (details on these considerations see [8]).

\section{Refugee Crisis Model}

The presented discrete model with transit regions allows modelling the pathway of refugees during the so-called Refugee Crisis 2015. Begin of September 2015 many people fled from war actions in Syria, and at the same time some of the refugee camps in Lebanon were shut down due to financial reasons - resulting in a refugee wave towards Western Europe. Figure 1 shows snapshots of data animation of refugees' pathway in the Refugee Crisis 2015 sketching the principle structure of the pathway. 


\subsection{Model graph structure}

For model implementation, the pathway is defined by a graph with nineteen vertices - the evident regions along the pathway. Origin is vertex $v_{1}$, region Syria, and final destinations are regions in Western Europe. It is not necessary - and because lack of data not possible - to investigate all countries on the pathway as self-consistent vertex, some countries are considered as common region along the pathway from Syria to Western Europe.

Table 1 lists the regions - the vertices implemented in the model, and Figure 5 shows the graph with the possibilities for migration.

\begin{tabular}{|c|c|}
\hline Vertices & Regions \\
\hline$v_{1}$ & Syria \\
\hline$v_{2}$ & Iraq \\
\hline$v_{3}$ & Jordan \\
\hline$v_{4}$ & Egypt \\
\hline$v_{5}$ & Lebanon \\
\hline$v 6$ & Turkey \\
\hline$v_{7}$ & Greece \\
\hline$v 8$ & Former Republic of Macedonia \\
\hline$v_{9}$ & Serbia \\
\hline$v_{10}$ & Hungary \\
\hline$v_{11}$ & Croatia \\
\hline$v_{12}$ & Slovenia \\
\hline$v_{13}$ & $\begin{array}{l}\text { Slovakia, Czech Republic, } \\
\text { Rumania, Bulgaria, Poland, } \\
\text { Lithuania, Estonia, Latvia }\end{array}$ \\
\hline$v_{14}$ & Austria \\
\hline$v_{15}$ & Germany \\
\hline$v_{16}$ & $\begin{array}{l}\text { United Kingdom, The Netherlands, } \\
\text { Belgium, France }\end{array}$ \\
\hline$v_{17}$ & Norway, Finland, Sweden, Denmark \\
\hline$v_{18}$ & Italy, Spain, Portugal \\
\hline$v_{19}$ & $\begin{array}{l}\text { Albania, Bosnia and Herzegovina, } \\
\text { Montenegro }\end{array}$ \\
\hline
\end{tabular}

Table 1. Regions - graph vertices - under consideration for Refugee Crisis model.

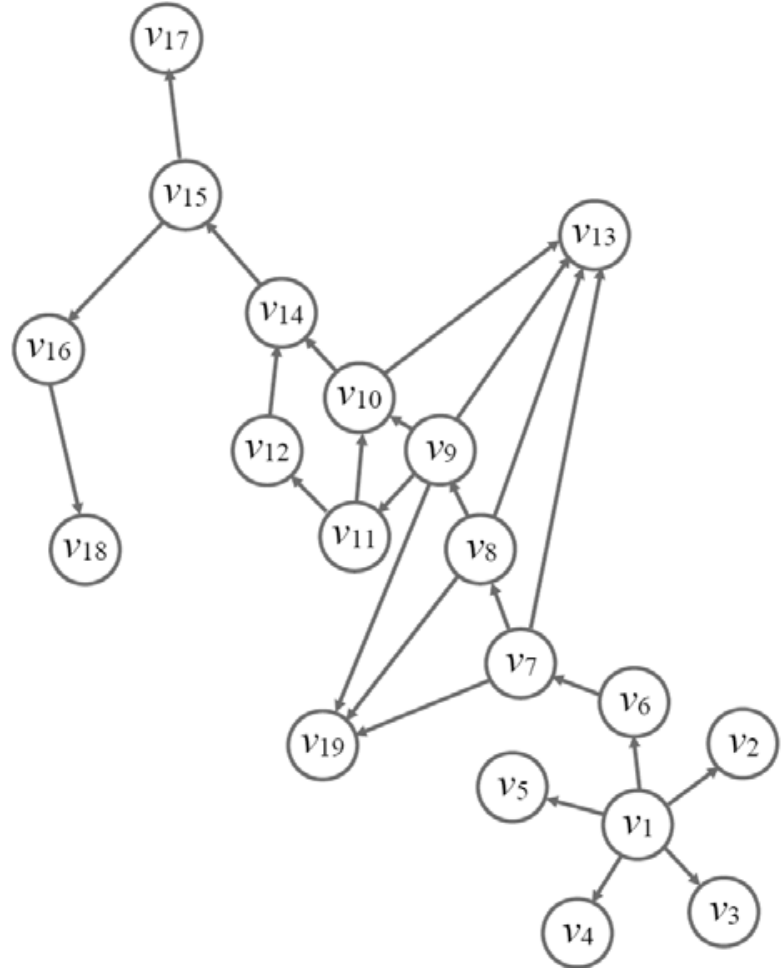

Figure 5. Migration graph model for Refugee Crisis 2015 from Syria into and within Europe in September/October 2015.

\subsection{Attribute functions}

The model focusses on the migration movement and the different migration routes and it is not modelling any reasons for emigration. This implies a distinction of countries of origin and potential destination countries. Observing the interactions from Syria to the neighbouring countries, the repulsive attributes of the country of origin are not taken into consideration. Here the attractive attributes of the neighbouring countries are determining the intensity of interaction.

Necessary is also a distinction between potential destination countries and transit countries. The attractive attributes of a transit country have less influence on the interaction; they are overlaid by the attraction attributes of the next destination regions. In the model, a transit region (country) is defined by a region, the repulsion attribute of which exceeds a certain limit. In this case, the attractive attributes of the region are replaced by an appropriate maximum of the attractive function of the countries, which are accessible from the transit region (formulas see Section 3.3). 
The choice of attraction attributes and repulsion attributes follows investigations and negotiations published in a study of the Australian economic scientist Timothy Hatton ([4]), and investigations by Neumayer on asylum destination choice ([5]). Hatton examined the correlation of specific attributes of developed countries and the number of asylum applications. As essential, he observed a big effect of access policy, like the border security, and of processing policy, like the humanitarian situation. Interestingly, the study shows only a small positive impact of welfare policy on the number of asylum applications ([4]). Table 2 and Table 3 list the seven attraction attributes and the five repulsion attributes used in this model implementation.

\begin{tabular}{cl}
\hline Function & Attraction Attributes \\
\hline $\boldsymbol{A}_{1, j}$ & Gross Domestic Product (GDP) \\
\hline $\boldsymbol{A}_{2, j}$ & Fragile State Index (FSI) \\
\hline $\boldsymbol{A}_{3, j}$ & Migrants in the country \\
\hline $\boldsymbol{A}_{4, j}$ & $\begin{array}{l}\text { Attractive attributes of accessible } \\
\text { countries }\end{array}$ \\
\hline $\boldsymbol{A}_{5, j}$ & Not exceeded capacity \\
\hline $\boldsymbol{A}_{6, j}$ & Asylum recognition rate in country \\
\hline $\boldsymbol{A}_{7, j}$ & Asylum recognition quote in Europe \\
\hline
\end{tabular}

Table 2. Seven attraction attributes for countries (regions) along pathway, $\mathrm{j}=1, . .19$.

\begin{tabular}{cl}
\hline Function & Repulsion Attributes \\
\hline $\boldsymbol{R}_{\mathbf{1}, j}$ & Gross Domestic Product (GDP) \\
\hline $\boldsymbol{R}_{\mathbf{2}, j}$ & Fragile State Index (FSI) \\
\hline $\boldsymbol{R}_{\mathbf{3}, j}$ & Exceeded capacity \\
\hline $\boldsymbol{R}_{\mathbf{4}, j}$ & Asylum recognition rate in country \\
\hline $\boldsymbol{R}_{\mathbf{5}, j}$ & Asylum recognition quote in Europe \\
\hline
\end{tabular}

Table 3. Five repulsion attributes for countries (regions) along pathway, $\mathrm{j}=1, . .19$.

The access policy and access control of a country is described by appropriate separation functions $C_{i, j}(t)$. In case of (partly) closed borders or closed borders enforced by border security arrangements, the separation functions reduce interaction immediately.
In general, time base for migration movement can be different - decades, years, quarters, months, and days. The investigated migration waves last one to three months, and data are available for the Refugee Crisis 2015 on a daily base, so a time base of days is appropriate for the model to be implemented. Clearly, the time base scales the interaction function $I_{i, j}$, and indirectly all attribute functions.

The unknown parameters in the model are the weighting parameters $a_{r}, r=1, \ldots, 7$ and $r_{s}, s=1, \ldots, 5$ in the formulas for the interaction function $I_{i, j}$. The parameters may differ for some countries, especially for the country of origin; some can be estimated, some must be identified using the data reported from UNHCR.

Figure 6 and Figure 7 show examples for the weighting parameters of attractive and repulsive attributes in classical destination countries. Of course, the attractive attributes of the county of origin must follow an exclusive non-attractive weighting - Figure 8.

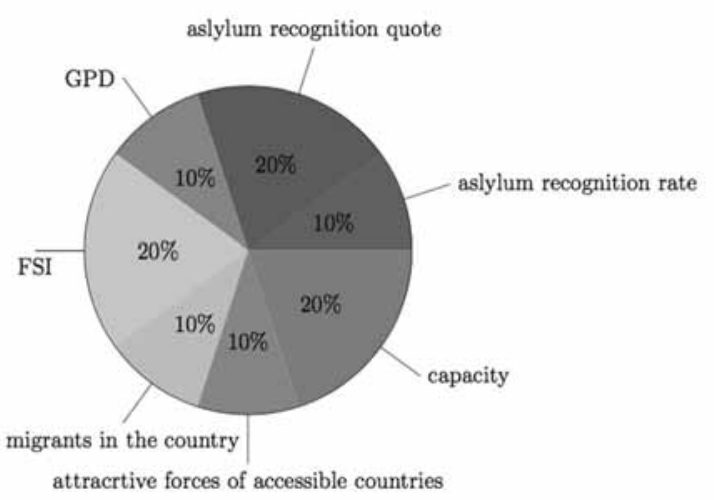

Figure 6. Weighting parameters for attractive attributes potential destination country.

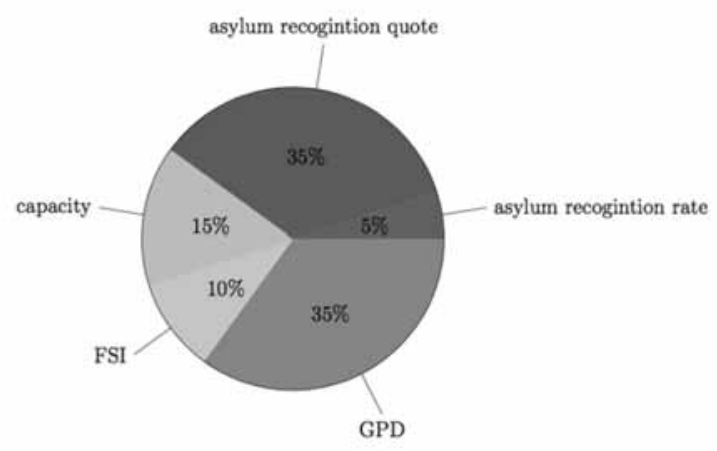

Figure 7. Weighting parameters for repulsive attributes potential destination country. 


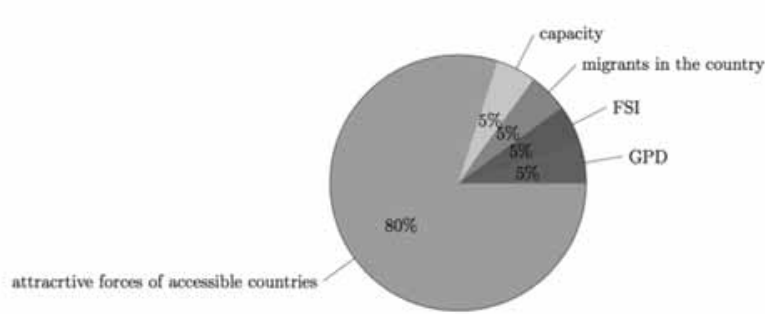

Figure 8. Weighting parameters for attractive attributes country of origin

\subsection{Software implementation}

The model description with the sum formulas and with the multiple indices looks difficult to implement. However, the graph structure with vector of vertices and adjacency matrix, and the possibility to formalize the attributes as vectors allows a compact model implementation in MATLAB language - code snippet in Figure 9.

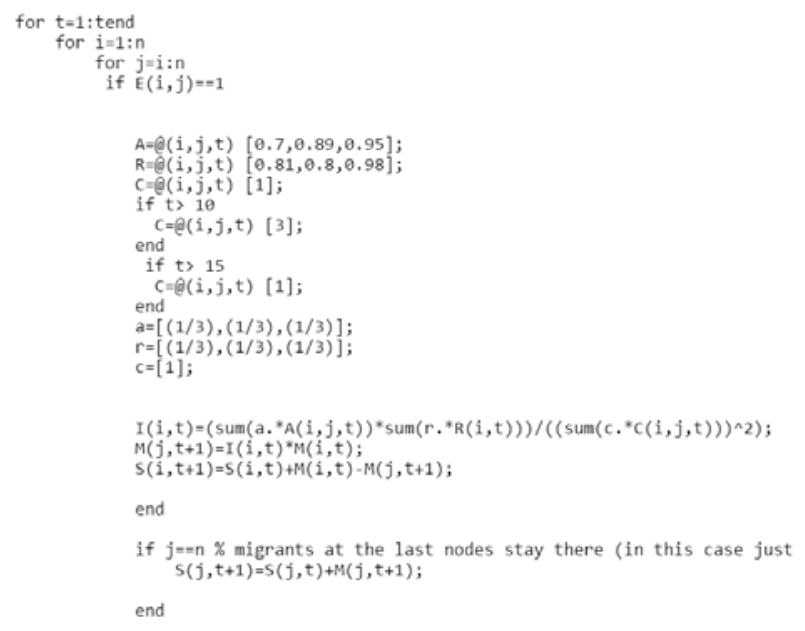

Figure 9. Model implementation in MATLAB - vector and matrix structures within main loop.

\subsection{Simulation results}

The implemented model allows a simulation of the pathway of refugees during the Refugee Crisis 2015, in the period from September 1, 2015 to October 31, 2015. A big challenge was the proper choice of the parameters the weighting parameters for the attributes. Some of them can be determined by available data, some must be identified in comparing UNHCR data from daily migrant movement with results from the simulation.

Most of the attraction attributes and repulsion attributes can be seen constant during these 61 days. At the first glance, the migration movement seems to be linearly growing over time, as shown with the number of refugees in the regions over time (Figure 10).

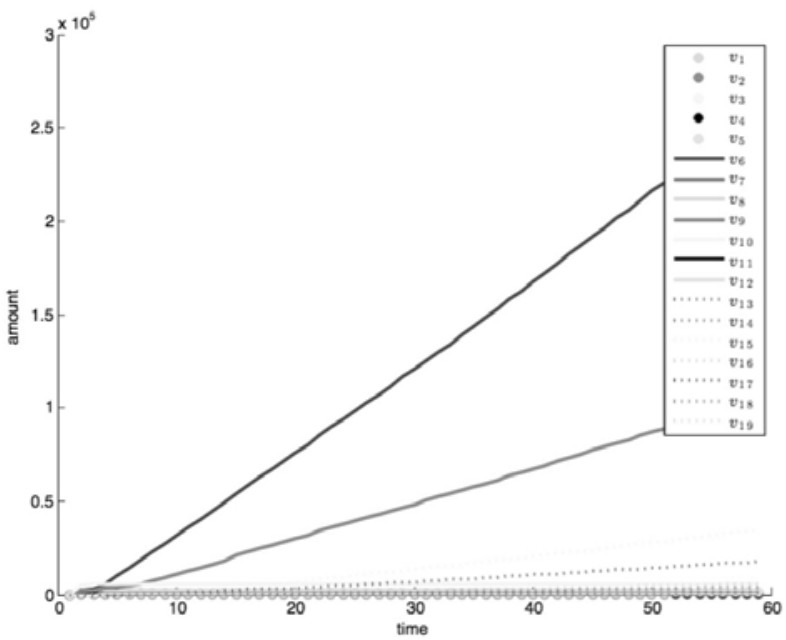

Figure 10. Result from simulation of Refugee Crisis 2015: number of refugees passing the 19 regions - almost linear growing behaviour.

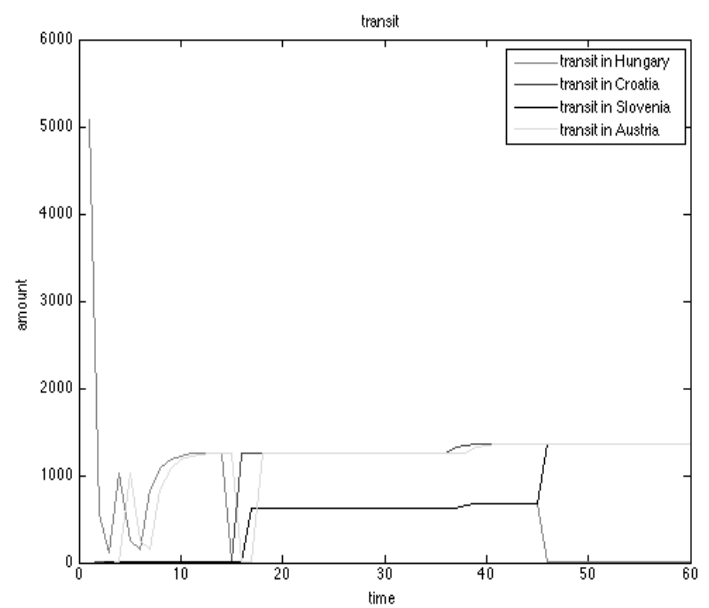

Figure 11. Result from simulation of Refugee Crisis 2015: number of refugees passing Hungary, Croatia, and Slovenia under impact of closing of borders on September 15 and 16, 2015.

The separation attributes clearly are not constant, they reflect the closing of the border from Hungary to Serbia on September 15, 2015, and the closing of the border from Hungary to Croatia on October 16, 2015, and subsequent opening of borders. Figure 11 impressively demonstrates the effect of the closing of borders on the amount of refugees trying to pass these borders. Clearly, under these circumstances the linear growth is replaced by constant values (and zero values) for migration.

The quality of the results can be checked by comparison with UNHCR data, at best with the summed-up number of asylum applications in each region. 


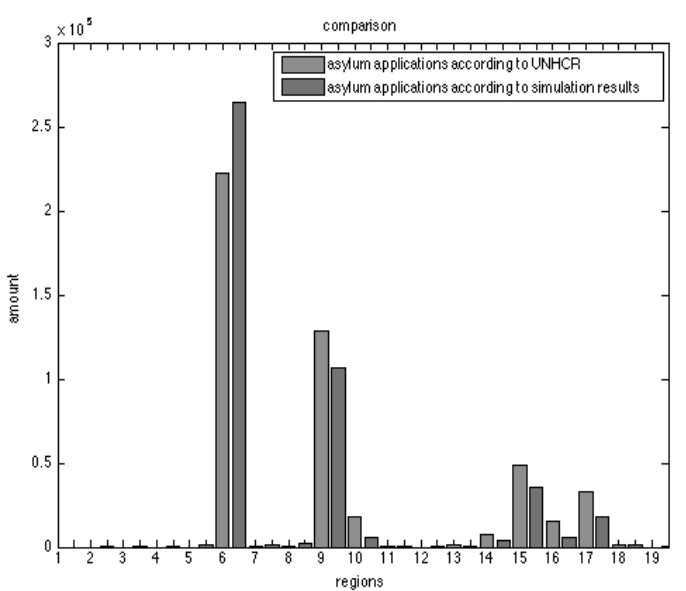

Figure 12. Result from simulation of Refugee Crisis 2015: summed up number of asylum applications in the 19 regions (right column, blue) compared with UNHCR data (left column, green) at the end of the refugee wave.

Simulation results in Figure 12 show the results of the migration movements as summed up number of asylum applications (during the full period) in the nineteen regions and compares these results with data recorded by UNHCR.

Some of the numbers in Figure 12 seem to be astonishing, as many people expect the highest number of asylum applications in Western Europe. However, the highest number of applications were put in vertices $v_{6}$ and $v_{9}$ - Turkey and Serbia. Significantly lower are the applications in the vertices $v_{15}, v_{17}, v_{16}$ and $v_{14}$-Germany, Scandinavia, France/UK, and Austria.

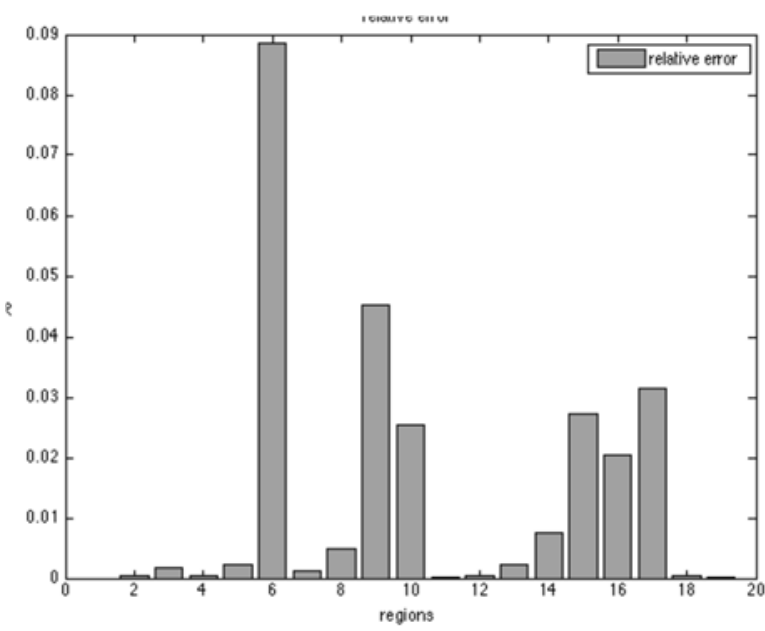

Figure 13. Result from simulation of Refugee Crisis 2015: relative error for summed up number of asylum applications in the 19 regions (simulation results data vs. UNHCR data).
The comparison of data from simulation and UNHCR data for the summed-up number of asylum applications allows calculating a relative error for the simulation result data, displayed in Figure 13. For most regions, the relative error is below $5 \%$, only for vertex $v_{6}$ - Turkey - it goes up to $9 \%$. Such relative errors are not satisfying for simulations of technical systems, but for socio-economic systems these errors are acceptable and lower than expected.

\subsection{Animation of simulation results}

Time plots can present results of the simulation, e.g. the number of migrants, as with Figure 10 and Figure 11, or bar charts with summed-up number of asylum applications. However, it is challenging to display also the pathways. Here, an animation of this specific refugee wave was implemented by $d w h$ Simulation Service for ORF 1 Online (Austrian Broadcast Company, Web Science Program Online).

The web-based animation with a sequence of 61 maps of Europe show for each of the 61 days

- the pathway of migrants by arrows from regions to region (the thickness of the arrow indicates qualitatively the up-to-data summed-up number migrants,

- the summed-up number of transit migrants and migrants applying for asylum in each region,

- and border actions by prohibitory signs at restricted or closed borders.

Figure 1 already presented two snapshots of this animation, but data driven. Now this animation can be driven by simulation data: Figure 14 and Figure 15 show snapshots, the one from begin of the refugee wave, and the other from end of wave, after the closing of borders.

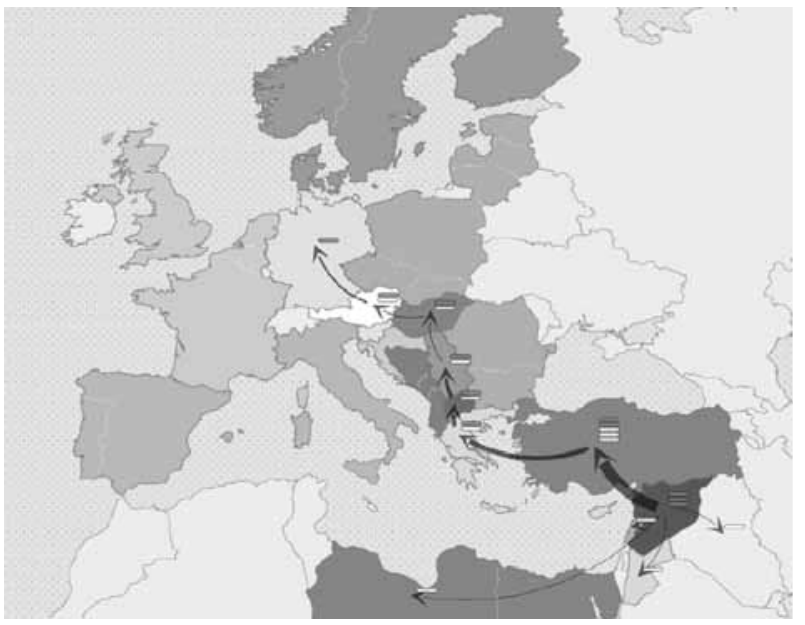

Figure 14. Refugee Crisis 2015: Simulation data animation, snapshot from begin of refugee wave.

SNE 27(4) - 12/2017 


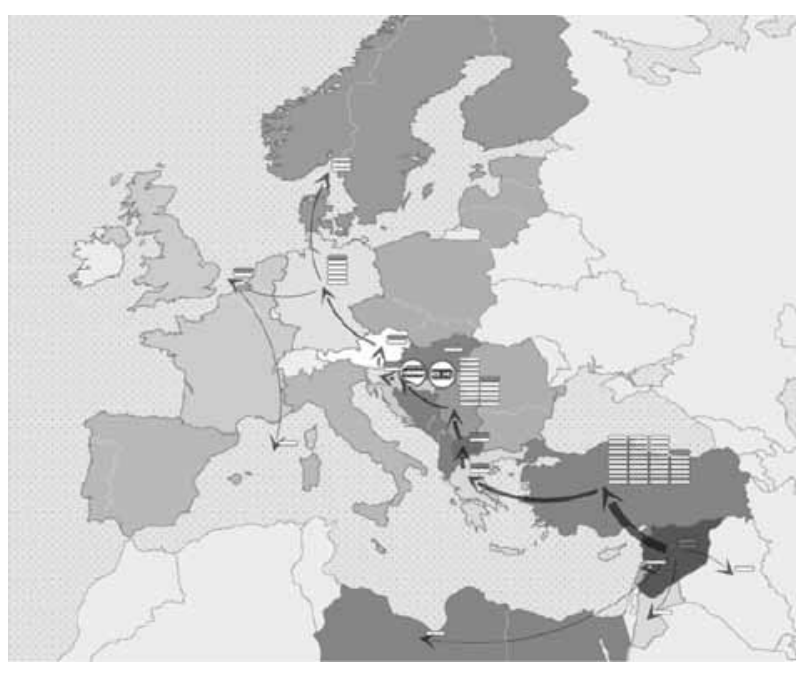

Figure 15. Refugee Crisis 2015: Simulation data animation, snapshot from end of refugee wave, with border closing at Balkan route.

\section{Forecast Model}

The Refugee Crisis Model allows an a-posteriori simulation of the Refugee Crisis 2015: the model was developed after the refugee wave, and the simulation results could be verified by data reported during the refugee wave. As the value of modelling and simulation is mainly forecast of dynamic behaviour, the question arises, whether the model can simulate a pathway of migration in advance a need for a Forecast Model.

In spring 2016, the so-called Balkan Route was closed by border control and by the so-called Turkey Deal. Nevertheless, for June 2016 again a refugee wave was expected, using the Mediterranean route. The challenge was to forecast parts of this wave by simulation, using a modification and extension of the Refugee Crisis Model.

The main changes for the Forecast Model are

- change of regions

- change of pathways, and

- change of some attributes.

Main task was the implementation of the Mediterranean route. An easy and generic solution is to continue the already existing pathway to Africa and connect it with the northern Mediterranean coasts. As consequence, vertex $v_{4}$ - Egypt - changes to vertex $v_{4}$ - North Africa/Coast, and the sea route is defined by a new pathway from vertex $v_{4}$ - North Africa/Coast to vertex $v_{18}$ - Italy, Spain, Portugal.

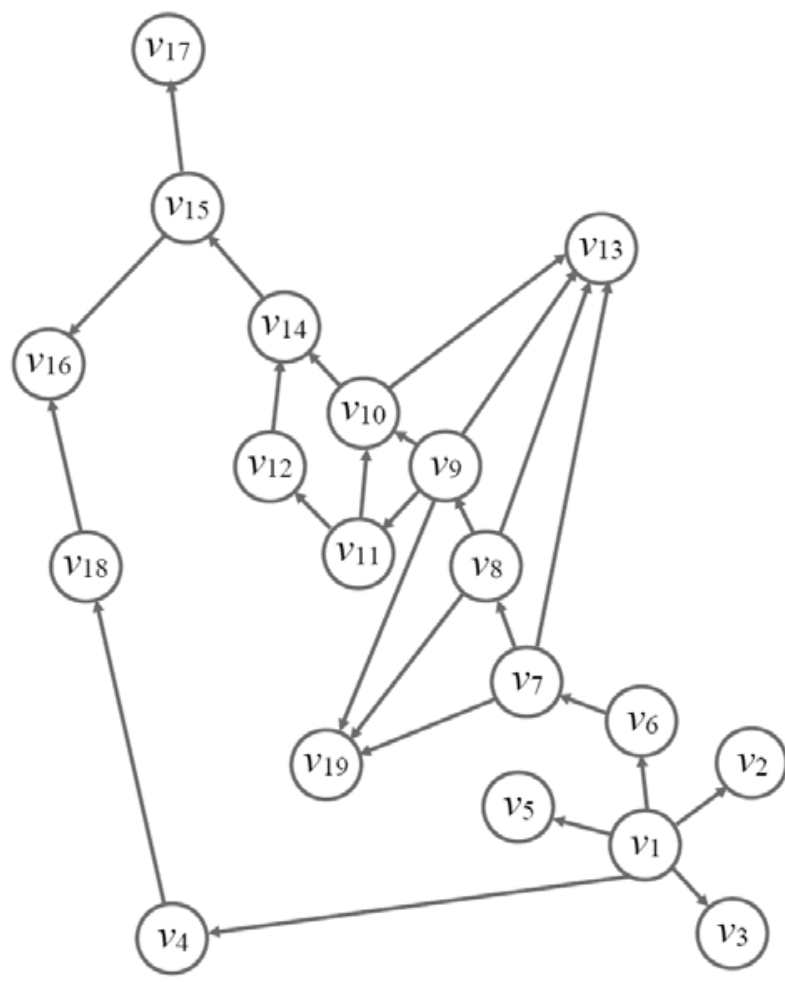

Figure 16. Migration graph model for Forecast Model with Mediterranean Sea route.

Figure 16 shows the new migration graph, with pathway along the Mediterranean Sea Route. It is not necessary to take into account a pathway from vertex $v_{4}$ - North Africa/Coast to vertex $v_{7}$ - Greece-because of the Turkey Deal, (as with the pathway to Balkan coast, because of the closed Balkan Route).

The Forecast Model can re-use most of the parameters and values of the attributes, except separation attributes, and some attraction attributes. The separation attributes $C_{i, j}$ of regions at Balkan model a closure of the borders for migrants, and separation attributes in Middle Europe (vertices $v_{15}, v_{17}$ - Germany, Scandinavia,) are increased (stronger border control for migrants). Furthermore it is assumed, that vertex $v_{16}-$ Western Europe has exceeded the asylum capacity (lower attraction attributes).

The model is parametrized to forecast by simulation the pathway of migrants and the number of asylum application for June 2016, with the same time base as the previous model. The simulation results calculated before the June 2016 migrant wave can now be compared with available data collected during and at the end of June 2016 migrant wave - displayed in barcharts for the asylum applications in Figure 17. 


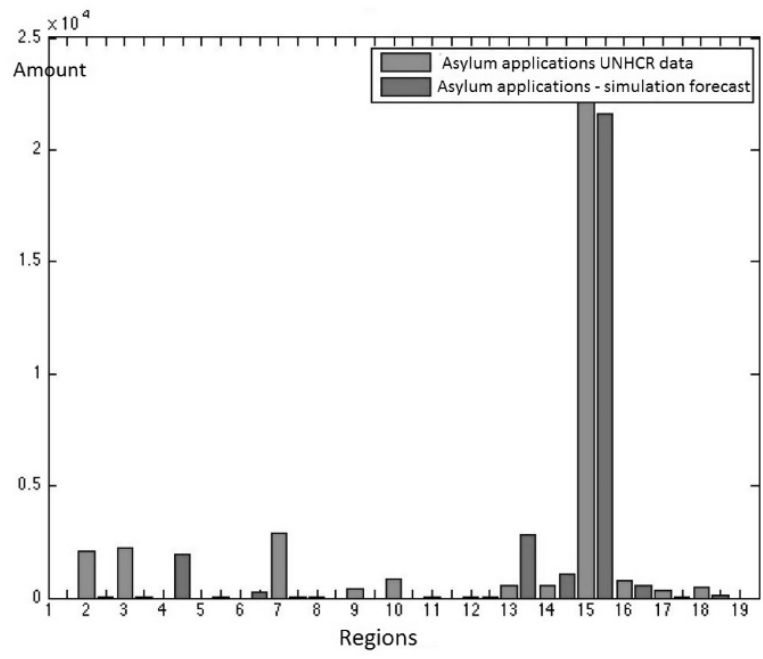

Figure 17. Forecast Model - June 2016 Migrant Wave summed up number of asylum application in the 19 regions (right column, blue) compared with compared with UNHCR data (left column, green) at the end of the refugee wave.

The simulation results are partly sufficient. Migrants put most asylum applications in vertex $v_{15}$ - Germany, here simulation results coincide well with a-posteriori data (8\% relative error). Satisfying are also results for $v_{16}-$ Western Europe (15\% relative error) and $v_{14}$ - Austria (26\% relative error). The significant higher relative errors for Balkan and Mediterranean regions are partly based on the fact, that data were not available, so that average data from spring 2016 were used. An exception is vertex $v_{7}$-Greece: the high number of real applications seems to include applications from migrants, who arrived earlier, and who had to stay because of the closure of the Balkan Route.

The relative errors of parts of the results of the Forecast Model are high. Such relative errors are not satisfying for simulations of technical systems, but for socioeconomic systems these errors are acceptable, especially they still allow a qualitative analysis of the dynamic behaviour.

Indeed the power of the Forecast Model is the possibility of a qualitative forecast of the migration pathway, especially when displayed in ascertainable manner for time domain, spatial domain, and flow domain, as with the web-based animation of maps of Europe with dynamic migration. Figure 18, Figure 19, and Figure 20 show three snapshots from this animation, the first at begin of June with closed borders, the second midst of June with already frequently used Mediterranean Sea route, and the third end of June, with final data.

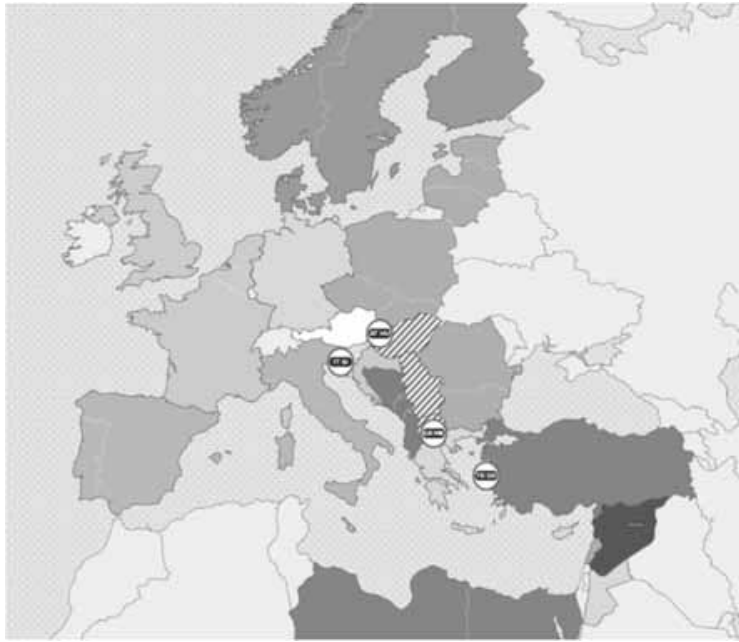

Figure 18. Forecast Model - Refugee Wave June 2016: simulation data animation, snapshot at begin.

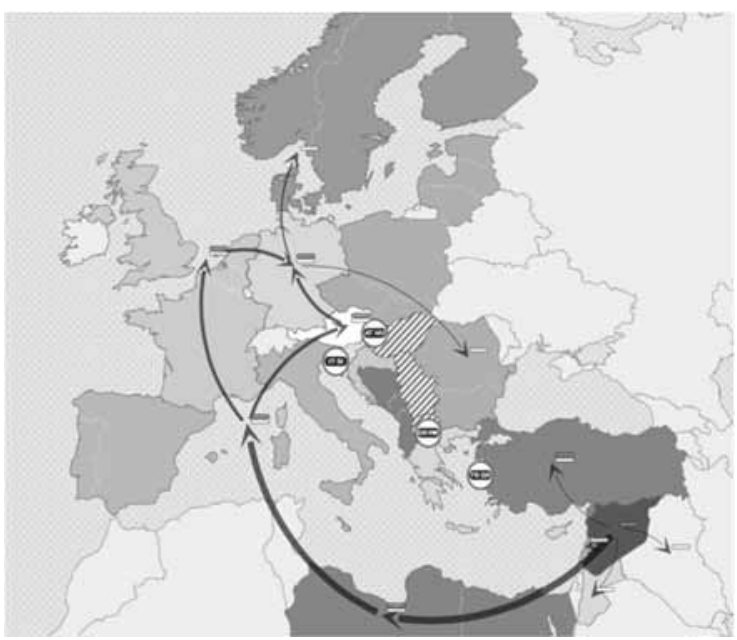

Figure 19. As Fig. 18, snapshot midst of June, frequently used Mediterranean Sea Route.

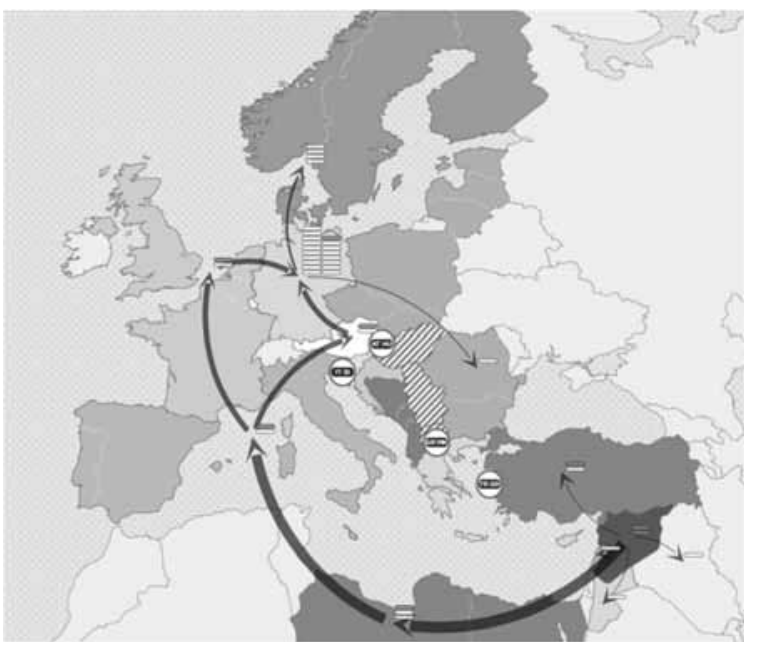

Figure 20. As Fig. 18, snapshot end of June, frequently used Mediterranean Sea Route and distribution of asylum applications. 


\section{Scenario Model}

Last step in the model development was the parametrization of the Forecast Model with average values for attraction attributes and for repulsion attributes, on basis of the structure with 19 regions. Additionally, this model provides some special separation attributes in order to study certain separation control, among them the closing of the Brenner border, the closing of German borders, etc.

This model allows analyzing qualitatively these scenarios by means of the barcharts for asylum application, and by means of the animated maps of Europe with migration pathways. Figure 21 shows one of these scenarios: Balkan Route closed, German borders closed - the result is a very strong pathway along the Mediterranean Sea Route - with all side effects.

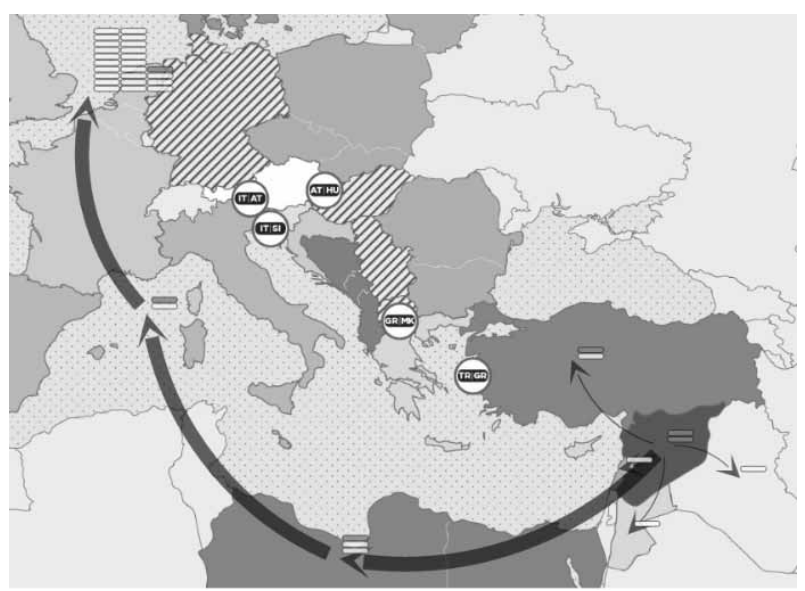

Figure 21. Scenario Model - Refugee Wave with extensive use of Mediterranean Sea Route.

\section{Conclusion}

'Models are in any case a simplification of reality, but they should help in better understanding of complex dynamics as migration movement, and the intention of this model is to show strategies to improve the situation of migrant population and resident populations under appropriate prerequisites.'

The above statement expresses the hope of the authors that the simulation really helps in understanding better pathways of migrants and refugees, and to think of the human beings involved. Clearly, the attributes in the regions control the flow of migrants. Still politicians think only on control by separation attributes (let's close the borders!), instead of thinking on an evenly distribution of attraction attributes in all regions, or on decrease of repulsion attributes (welfare, no wars, ...).
Many people think that figures with pathways of migrants along the Mediterranean Sea Route are a novelty in Europe (e.g. Figure 29 as reality, or Figure 21 as scenario). The main author found a very similar picture with pathways of migrants along the Mediterranean Sea Route (see below) in an article by J. Fischer about the migration of the sea people at the end of Bronze Age ([3]) - let's also learn from history!

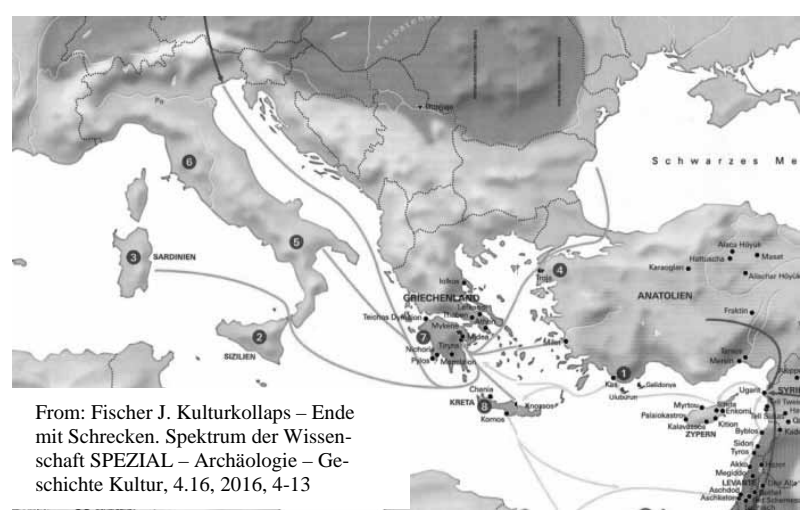

\section{References}

[1] Carey H C. Principles of Social Sciences. Philadelphia, Pennsylvania 1858.

[2] Dennett A. Estimating flows between geographical locations: get me started in spatial interaction modelling. Working Paper Series, Paper 181-March 12, UCL, Centre for Advanced Spatial Analysis, 2012.

[3] Fischer J. Kulturkollaps - Ende mit Schrecken. Spektrum der Wissenschaft SPEZIAL - Archäologie Geschichte Kultur, 4.16, 2016, 4-13.

[4] Hatton T, Maloney. Applications for Asylum in the Developed World: Modelling Asylum Claims by Origin and Destination. ANU Working Papers in Economics and Econometrics, 625, Australian National Univ. 2015.

[5] Eric Neumayer. Asylum Destination Choice: What makes some west European countries more attractive than others?, European Union Politics, Volume 5 (2), 155-180, SAGE Publications, 2004, London.

[6] Sen A, Smith T. Gravity Models of Spatial Interaction Behaviour. Springer, 1995.

[7] Vobruba T. Modellbildung, Analyse und Simulation eines räumlichen Interaktionsmodells. Master Thesis TU Wien, 2016, p.115.

[8] Vobruba T, Körner A, Breitenecker F. Modelling, Analysis and Simulation of a Spatial Interaction Model. Proc. 17th IFAC TECIS Conf., P. Kopacek, E. Hajrizi (eds); IFAC-PapersOnLine Volume 49, Issue 29, 221-225. doi 10.1016/j.ifacol.2016.11.054

[9] Young E C. The movement of farm population. Cornell Agricultural Experiment Station, New York, 1924. 